\title{
Cuidado de pessoas com feridas crônicas na Atenção Primária à Saúde
}

Care of people with chronic wounds in Primary Health Care

Cuidado de personas con heridas crónicas en la Atención Primaria de Salud

Natalia Maira Resende ${ }^{1}$, Tatiane Cínthia Nascimento ${ }^{2}$, Fellype Rodrigues Freitas Lopes ${ }^{3}$, Antônio Gilson Prates Junior ${ }^{4}$, Nathan Mendes Souza ${ }^{5}$

\section{Resumo}

Caracterizar e melhorar a qualidade do cuidado prestado aos pacientes portadores de feridas crônicas no âmbito da Atenção Primária à Saúde. Foi feito uma pesquisa-ação mediante implementação de estratégia multifacetada (i.e.; visitas domiciliares, capacitação da equipe, desenvolvimento e inserção de ficha no prontuário dos pacientes) para a melhoria da qualidade de serviços e do registro dos cuidados dos pacientes com feridas crônicas. Utilizou-se métodos de análise de prontuários e observação participante. Incluiu-se 17 pacientes com feridas crônicas, nove $(52,9 \%)$ com úlcera de origem venosa, dois $(11,7 \%)$ com úlcera de origem arterial, dois com úlcera decorrente de pé diabético, dois com úlcera de pressão, um com úlcera de origem neoplásica e um paciente com úlcera com etiologia mista. As visitas domiciliares evidenciaram discrepância do autocuidado entre os pacientes e sua relação direta no processo de cura das úlceras. Deficiente autocuidado parece influenciar o surgimento, cronificação e cura demorada de feridas crônicas e pode estar relacionado as baixas condições sócio-economica-culturais locais; precários hábitos de higiene, baixa literacia sobre feridas crônicas, baixo acesso aos serviços de saúde e frágil rede familiar e comunitária de apoio. Educação permanente dos membros da Estratégia Saúde da Família e inserção de instrumentos de avaliação de feridas crônicas podem melhorar a qualidade dos cuidados ofertados no âmbito da Atenção Primária a Saúde. Estratégias multifacetadas de melhoria de qualidade dos cuidados e registro de serviços prestados a pessoas com úlceras crônicas podem ser efetivas, mas necessitam ser apropriadamente monitoradas e avaliadas.

Descritores: Úlcera da Perna; Insuficiência Venosa; Assistência Domiciliar; Assistência a Idosos, Saúde da Família.

\footnotetext{
Abstract

To characterize and improve the quality of care provided to patients with chronic wounds in primary care. We performed an action-research by implementing a multifaceted strategy (i.e.: home visits, professional capacity building, development and inclusion of new instruments in patients' records), for quality improvement of the services and of its health recording for patients with

${ }^{1}$ Estudante do curso de medicina. Universidade Federal de Ouro Preto, R. Diogo de Vasconcelos, 122 - Pilar, Ouro Preto - MG, 35400-000, Brasil. E-mail: nataliamresende@yahoo.com.br

${ }^{2}$ Estudante do curso de medicina. Universidade Federal de Ouro Preto, R. Diogo de Vasconcelos, 122 - Pilar, Ouro Preto - MG, 35400-000, Brasil. E-mail: tatianenasc@yahoo.com.br

${ }^{3}$ Estudante do curso de medicina. Universidade Federal de Ouro Preto, R. Diogo de Vasconcelos, 122 - Pilar, Ouro Preto - MG, 35400-000, Brasil. E-mail: fellype_freitas@hotmail.com

${ }^{4}$ Estudante do curso de medicina. Universidade Federal de Ouro Preto, R. Diogo de Vasconcelos, 122 - Pilar, Ouro Preto - MG, 35400-000, Brasil. E-mail: antonio.ufop@gmail.com

${ }^{5}$ Mestre em Educação Médica pela Universidade Federal de Ouro Preto, médico de família e comunidade e docente do Departamento de Clínica Médica da Faculdade de Medicina da Universidade Federal de Minas Gerais. Endereço: Av. Prof. Alfredo Balena, 190 - Santa Efigênia, Belo Horizonte - MG, 30130-100, Brasil. Email: nameso@ufmg.br
} 
ISSN 2179-6750

chronic wounds. We audited patients' health records and undertook participant observation. We included 17 patients with chronic wounds, nine patients $(52,9 \%)$ with venous ulcer, two $(11,7 \%)$ with arterial ulcer, two with ulcers from diabetic foot, two with pressure ulcer, one with neoplasic ulcer and one with ulcer of mixed etiology. Home visits showed discrepancy of self-care among patients and its direct relation with the healing process. Deficient self-care seems to influence the onset, chronicity and delayed healing of chronic ulcers, and it may be related to local low socioeconomic and cultural conditions; poor hygiene, low literacy on chronic wounds, insufficient access to health service and frail family and community support network. Professional continuing education and inclusion of a tool to evaluate chronic wounds may improve quality of the services in primary care. Multifaceted strategy to improve the quality of care and the registry of services provided to people with chronic ulcers may be effective, but appropriate monitoring and evaluation are necessary.

Key-words: Leg Ulcer; Venous Insufficiency; Home Nursing; Old Age Assistance; Family Health.

\section{Resumen}

Caracterizar y mejorar la calidad de la atención prestada a los pacientes con heridas crónicas en el ámbito de la Atención Primaria en Salud. Se realizó una investigación-acción mediante la aplicación de estrategia multifacética (visitas domiciliarias, capacitación del personal, desarrollo y inserción de expediente en los registros médicos de los pacientes), para mejorar la calidad de los servicios y el registro de la atención a los pacientes con heridas crónicas. Se utilizaron métodos de análisis de registros médicos y la observación participante. Se incluyó 17 pacientes con heridas crónicas, nueve $(52,9 \%)$ con úlceras de origen venoso, dos $(11,7 \%)$ con úlcera de origen arterial, dos con úlceras del pie diabético, dos con úlcera por presión, uno con ulcera de malignidad y uno con úlcera de etiología mixta. Las visitas domiciliarias mostraron discrepancia de autocuidado entre los pacientes y su relación directa en el proceso de curación de las úlceras. El deficiente autocuidado parece influir en la aparición, la cronicidad y retraso en la cicatrización de las heridas crónicas y puede estar relacionado con las condiciones socio-económicas y culturales locales; la falta de higiene, la falta de información en heridas crónicas, bajo acceso a servicios de salud y la red familiar y de apoyo comunitario frágiles. La educación de los miembros de la Estrategia Salud de la Familia y la inserción de los instrumentos de evaluación de las heridas crónicas puede mejorar la calidad de la atención ofrecida en el contexto de la Atención Primaria de la Salud. Estrategias multifacéticas para mejorar la calidad de la atención y el registro de los servicios prestados a personas con úlceras crónicas pueden ser eficaces, pero deben ser supervisados y evaluados correctamente.

Palabras-claves: Úlcera de la Pierna; Insuficiencia Venosa; Asistencia a Domicilio; Asistencia a los Ancianos, Salud de la Familia.

\section{Introdução}

As feridas crônicas podem ser definidas como feridas de longa duração ou de reincidência frequente $^{1}$. Sua etiologia está associada a diversos fatores como: doença venosa crônica, doença arterial periférica, neuropatias, hipertensão arterial, trauma físico, anemia falciforme, infecções cutâneas, doenças inflamatórias, neoplasias e alterações nutricionais.

As feridas crônicas constituem grave problema de saúde pública no Brasil devido à alta morbidade e custos terapêuticos bem como pela diminuição da qualidade de vida associada. 
ISSN 2179-6750

Duração prolongada do tratamento, ocorrência de recidivas, necessidade de adesão do paciente ao tratamento $^{2}$ bem como resistência microbiana ${ }^{3}$ são elementos que contribuem para a grande morbidade relacionada às feridas crônicas.

Existe escasso e deficiente registro referente à epidemiologia (i.e., prevalência e incidência) e aos processos de cuidado das feridas crônicas (ex., etiologia, métodos de diagnóstico, monitoramento e tratamento, período de evolução, complicações, impacto na qualidade de vida e custos) no âmbito da Atenção Primária à Saúde (APS) no Brasil. Sua terapêutica efetiva envolve abordagem da condição de base e uso de medidas locais cicatrizantes ${ }^{2}$. Agravamento do quadro pode ocorrer mediante tentativas dos próprios pacientes de tratamento da ferida, muitas das quais baseadas em abordagens inapropriadas, geralmente recomendadas por familiares ou amigos 1 .

O cuidado da saúde dos indivíduos portadores de feridas é um problema de grandes dimensões representando um desafio a ser enfrentado cotidianamente, por quem vivencia tal problema, seus cuidadores e pelos serviços de saúde ${ }^{4}$. Atualmente, busca-se empoderar pessoas suscetíveis ou com feridas crônicas instaladas na prevenção e auto-cuidado deste agravo. A educação em saúde exerce importante influência nas mudanças nos hábitos de vida e para a adesão ao tratamento clínico ${ }^{1}$. O objetivo deste estudo é caracterizar e melhorar a qualidade do cuidado prestado aos pacientes portadores de feridas crônicas no âmbito da Atenção Primária à Saúde.

\section{Método}

Trata-se de uma pesquisa-ação realizado entre janeiro e agosto de 2014 mediante implementação de estratégia multifacetada (i.e.; visitas domiciliares, capacitação da ESF, desenvolvimento e inserção de ficha no prontuário dos pacientes) para a melhoria da qualidade de serviços e do registro dos cuidados dos pacientes com feridas crônicas. Utilizou-se métodos de análise de prontuários e observação participante para recoleção de dados. Como processo de desenvolvimento ou melhoria da qualidade do serviço prestado na APS, os autores deste manuscrito entendem existir dispensa da aprovação de consentimento de Comitê de Ética de Pesquisa. Segundo Eccles et al (2011) $)^{5}$, alguns autores descrevem seu trabalho como melhoria da qualidade (ou algum serviço denotando atividades em desenvolvimento, em vez de pesquisa), o que não exigiria revisão do comitê de ética.

No período de janeiro a março de 2014, elaborou-se lista dos pacientes portadores de feridas crônicas com médicos, enfermeiros, técnicos de enfermagem e agentes comunitários de saúde (ACS). Auditorou-se os prontuários destes pacientes para aferir a qualidade do registro. Fizeram-se visitas domiciliares aos pacientes e suas famílias. Desenvolveu-se ficha para avaliação de feridas 
ISSN 2179-6750

(Figura 1) mediante revisão da literatura e diálogo com os profissionais de saúde da Estratégia Saúde da Família (ESF) da Unidade de Atenção Primária à Saúde CAIC (UAPS-CAIC), em Cachoeira do Campo, Ouro Preto (Minas Gerais), com o intuito de registrar dados referentes ao histórico da ferida, comorbidades, fatores de risco para cronificação da ferida, localização e características da mesma. Confeccionou-se folheto com orientações gerais de prevenção de feridas (Figura 2) para distribuição aos pacientes predispostos a desenvolver feridas crônicas segundo perfil sanitário da UAPS e confirmado pelas ACS (ex.: pacientes com diabetes, dislipidemia e acamados). Entre julho e agosto de 2014, todos os pacientes foram revisitados e os prontuários reauditorados, para avaliar a evolução das feridas, qualidade do seguimento e do registro.

\section{Resultados e Discussão}

\section{Caracterização da UAPS CAIC}

A UAPS CAIC está localizada em zona urbana do distrito de Cachoeira do Campo, pertencente ao município de Ouro Preto, Minas Gerais e abrange duas equipes da Estratégia Saúde da Família (ESF). A ESF Nova Aliança, com quatro anos de criação, atende 3130 pessoas (812 famílias) e a ESF Vida tem 13 anos e atende 2961 pessoas (771 famílias). Cada equipe é composta por um médico, um enfermeiro, um técnico de enfermagem e três agentes comunitários de saúde (ACS) na ESF Vida e quatro ACS na ESF Nova Aliança. A unidade conta também com um recepcionista, um auxiliar de serviços gerais e uma equipe do Núcleo de Apoio à Saúde da Família (NASF) composta por um terapeuta ocupacional, dois educadores físicos, um fisioterapeuta, um psicólogo, um nutricionista e um assistente social.

A região adstrita apresenta a maior parte das ruas asfaltadas e 95,85\% tem esgoto no domicílio. Apenas 3,14\% não tem água tratada em seus domicílios e há um risco ambiental que é a presença de voçorocas em todo território. Quanto ao perfil de morbidades prevalentes na população e relevantes para o estudo têm-se $752(12,35 \%)$ pessoas com hipertensão arterial sistêmica (HAS) e $206(3,35 \%)$ pessoas com diabetes mellitus (DM).

\section{Auditoria dos prontuários}

Mediante auditoria dos prontuários dos pacientes com alto risco de desenvolvimento de feridas crônicas e abordagem dos profissionais da UAPS-CAIC, identificou-se 18 pacientes com feridas crônicas. Um dos pacientes foi posteriormente excluído do seguimento porque mudou-se do território de abrangência e o prontuário não foi localizado. Os dados referentes às características do 
ISSN 2179-6750

paciente, qualidade do registro, características das úlceras e uso da UAPS constam na Tabela 1. A idade média destes pacientes foi de 64 anos, sendo 58,8\% (10/18) do sexo masculino. Dentre as comorbidades predominantes, destacam-se: HAS em 94,1\% dos pacientes $(n=16)$; diabetes melittus tipo 11 em 58,8\% ( $n=10)$; dislipidemia em 52,9\% ( $n=9)$; tabagismo em 35,2\% ( $n=6)$ e etilismo em 17,6\% ( $\mathrm{n}=3)$. Outras comorbidades: insuficiência cardíaca congestiva, doença pulmonar obstrutiva crônica, hipotireoidismo, artrite reumatóide, fibrilação atrial, bexiga neurogênica, glaucoma, carcinoma espinocelular, epilepsia e filariose. Não foi possível avaliar o tempo médio de evolução das feridas devido à escassez de dados no prontuário e desconhecimento pelo próprio paciente. No entanto, o tempo mínimo foi quatro meses e o máximo 30 anos. Também não foi possível caracterizar as feridas crônicas (i.e.: dimensões e aspecto das feridas). Nove $(52,9 \%)$ pacientes tinham úlcera de origem venosa, dois $(11,7 \%)$ pacientes tinham úlcera de origem arterial, dois pacientes com úlcera decorrente de pé diabético, dois pacientes com úlcera de pressão, um paciente com úlcera de origem neoplásica e um paciente com úlcera com etiologia mista (arterial e venosa). Ocorreu pelo menos um episódio de infecção da ferida entre agosto de 2013 e agosto de 2014 em $58,8 \%$ dos pacientes. O número máximo de infecções nos últimos 12 meses por paciente foi quatro.

De uma forma geral, os prontuários apresentam imprecisão de informações e ausência de registros referentes a caracterização das feridas, dos tratamentos utilizados e dos custos relacionados aos cuidados das feridas. Do total de prontuários, 23,5\% (n=4) não faziam referência à existência da úlcera. Oitenta e dois por cento da amostra apresentava folha de rosto, 70\% apresentava lista de problemas e 64,7\% apresentava registro orientado ao problema. Entre agosto de 2013 e agosto de 2014, 14 (82,3\%) foram atendidos pelo menos uma vez por um dos médicos da UAPS e 10 pacientes foram atendidos pelo menos uma vez por profissionais da enfermagem. Neste mesmo período, os 17 pacientes participaram de 68 consultas médicas, uma média quatro consultas por paciente sendo que um único paciente recebeu 13 consultas médicas. Houve 83 atendimentos pela enfermagem nos últimos 12 meses, com média de 4,8 atendimentos por paciente sendo que o máximo de atendimentos por profissionais da enfermagem para um mesmo paciente foi 25 .

Estudo de Vu et al (2007) ${ }^{6}$ realizado com idosos institucionalizados na Austrália encontrou relação entre a melhoria do cuidado e a chance de cicatrização de feridas. Este estudo demonstrou também que treinamento dos profissionais para lidar com as úlceras mostrou-se bastante eficaz e de baixo custo. Sanada et al $(2010)^{7}$ reiteraram a importância do treinamento da equipe cuidadora destes pacientes e da otimização dos recursos usados no tratamento de úlceras de pressão.

Visitas domiciliares 
ISSN 2179-6750

Entre agosto de 2013 e agosto de 2014 foram realizadas 75 visitas domiciliares por médicos e 89 visitas por profissionais da enfermagem aos pacientes com úlceras crônicas. A média de visitas por paciente realizadas por médicos foi de 4,4 e 5,2 pela enfermagem. O número máximo de visitas por pacientes foi de 18 por médicos e 23 pela enfermagem.

Observação participante dos autores deste estudo durante as visitas domiciliares evidenciaram a discrepância do autocuidado entre os pacientes e sua relação direta no processo de cura. Pacientes com melhor renda usam, por conta própria, materiais mais eficazes no tratamento de feridas, uma vez que o município não os oferece regularmente. Deficiente auto-cuidado parece influenciar o surgimento, cronificação e cura demorada de feridas crônicas e pode estar relacionado as baixas condições sócio-economica-culturais das pessoas, de suas família e de sua comunidade; precários hábitos de higiene, baixa literacia sobre feridas crônicas, precário acesso aos serviços de saúde (recursos humanos e insumos para tratamento) e pobre rede familiar e comunitária de apoio.

Observou-se ainda durante as visitas domiciliares que o tema "feridas" possui certos mitos relacionados ao processo de cura, como a recorrente frase: "se a ferida fecha, o coração para", e coloca, em alguns casos, o indivíduo na posição de "paciente", o que pode trazer ganhos pessoais secundários. Este conhecimento popular contrapõe-se ao conceito biomédico de adesão consciente e ativa ao plano de cuidados acordado entre a equipe de saúde e os paciente e seus familiares. Tais conhecimentos populares podem contribuir com a explicação de certas ações por parte dos paciente, que são vistas por alguns membros da ESF como impedidoras para a cicatrização e cura das feridas.

\section{Intervenções}

Diante da evidência de deficiente registro nos prontuários dos pacientes e do precário autocuidado observado nas visitas domiciliares, foi desenhado e implementado programa de capacitação de toda a ESF referente a prevenção, avaliação, tratamento e registro dos casos por meio de educação continuada. Realizou-se três encontros de educação permanente com médicos, enfermeiros, técnicos de enfermagem e agentes comunitários de saúde onde foram abordadas, através de apresentação dialogada de slides, caracterização de feridas quanto a fase de cicatrização, etiologias, abordagem e materiais usados em cada fase, bem como a prevenção das mesmas.

Trabalhou-se as Figuras 1 e 2; exibiu-se fotos de feridas (obtidas na internet e das feridas dos próprios pacientes da UAPS) em diversos estágios de evolução e vídeos de como abordar tais pacientes e seus familiares. Consensuou-se a utilização das fichas para avaliação de feridas como folha de rosto dos prontuários de saúde dos 17 pacientes, o que foi seguido por impressão e anexação destas aos respectivos prontuários. Tal iniciativa visou otimizar o monitoramento destes 
ISSN 2179-6750

pacientes em sua integralidade e na especificidade de suas feridas crônicas.

Figura 1. Formulário de avaliação de feridas

AVALIAÇÃO DE FERIDAS

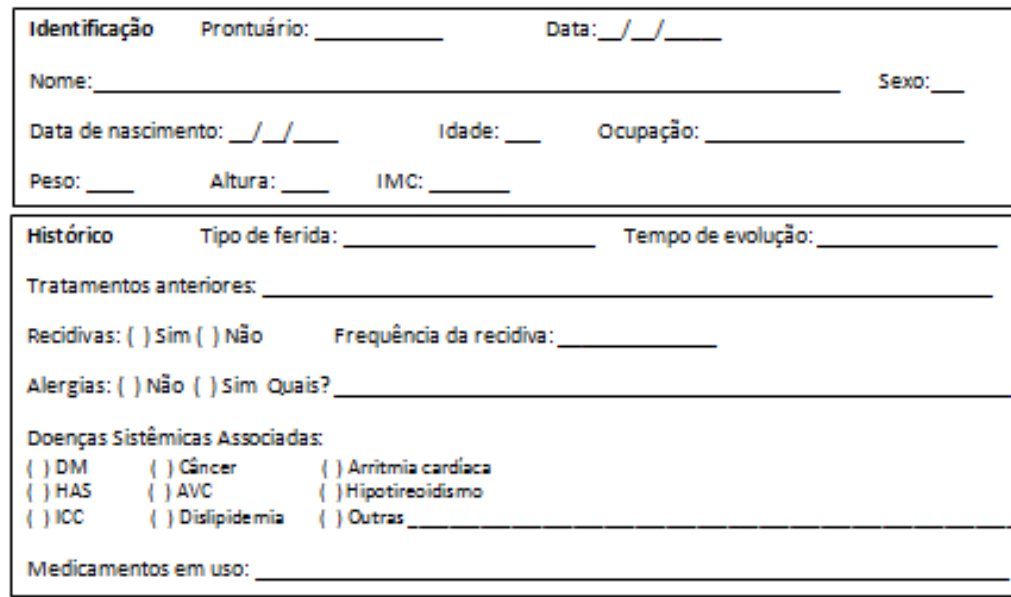

Fatores de risco para cronificação da úlcera

Tabagismo: ( ) Sim [ ] Näo obs:

Etilismo: ( ) Sim [ ] Nào Obs:

Desnutrição: [ ] $\operatorname{sim}$ [ ] Não

Acompanhamento com nutricionista? [ ] sim ( ) Näo

Higiene: [ ] Boa (] Regular ( ) Ruim [ ] Péssima

Mobilidade: ( ) Independente (]) Parcialmente Dependente () Cadeira de rodas (] Acamado

Capacidade de auto-cuidado: ( ) Otima () Boa () Ruim (] Precária
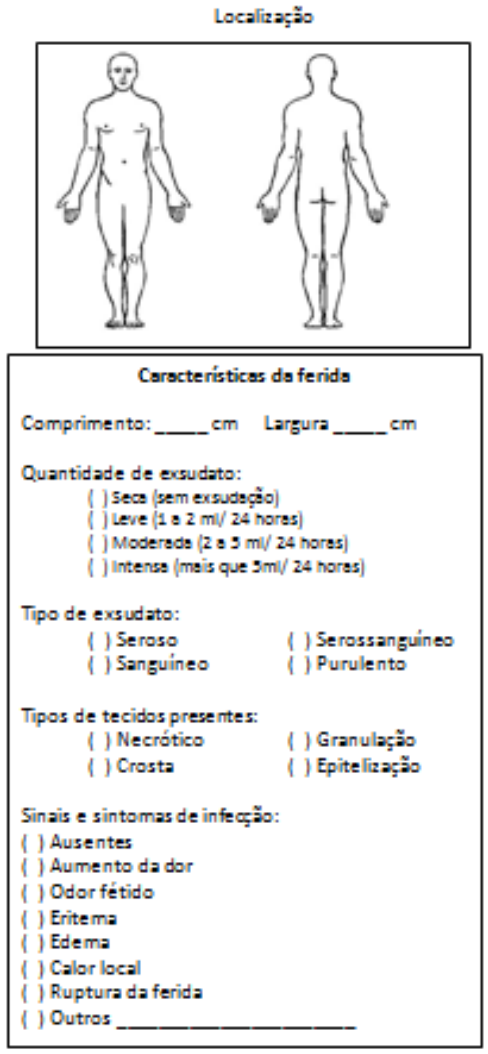

Figura 2. Folheto informativo direcionado aos pacientes em risco de desenvolver feridas crônicas

Pequenas atitudes podem ajudar na prevenção de feridas em membros inferiores. Cuide-se!

- Realize a higiene dos pés adequadamente: limpeza e secagem dos espaços entre os dedos e cortes de unhas retas - Hidrate bem sua pele

-Não ande descalço

-Examine diariamente seus pés verificando sinais de pré-

úlcera: bolhas, aumento da temperatura, hematomas, calosidades com fissuras, pontos avermelhados, inchaço localizadoe pontos dolorosos à palpação

- Não utilize sapatos novos por periodos prolongados

- Use meias macias, como de algodão, tendo o cuidado de

observar os pontos de costura que podem funcionar como

pontos de pressão. Recomenda-se, para prevenir áreas de atrito, o uso de meias no avesso

- Examine diariamente o interior dos calçados: procure. pontos endurecidos, pregueamento da palmilha ou presença de objetos estranhos

Em caso de alguma anormalidade, procure seu médico! As

Glceras dos membros inferiores sa prode dif́cile demorado

tratamento e você pode evitá-las. 
ISSN 2179-6750

Uma dificuldade por parte de todos os profissionais de saúde na abordagem das feridas é o reconhecimento de variáveis pertinentes ao cuidado. Uma importante etiologia é representada pelas úlceras venosas. De acordo Tramontini ${ }^{8}$, em um estudo realizado no Brasil, com 1755 pessoas acima de 15 anos, foi encontrada uma prevalência de 3,6\% de úlceras venosas ativas e/ou cicatrizadas, das quais $2,3 \%$ ocorreram em homens e $4 \%$ ocorreram em mulheres. Os profissionais devem ser capacitados para avaliar características como o tamanho da ferida, o local de ocorrência, margens, presença de exsudato, edema, hiperpigmentação, dor, presença de dermatite associada, pulsos, doença avançada, esfacelo e evolução da ferida ${ }^{8}$.

Seguimento dos pacientes

Três meses após o processo de educação permanente visando melhoria da qualidade dos serviços ofertados pelos profissionais do UAPS CAIC, os pacientes foram revisitados e nova auditoria dos prontuários foi realizada. Um paciente havia falecido por complicações da doença de base. Em cinco pacientes $(5 / 17=29,4 \%)$ a úlcera havia cicatrizado, sendo dois pacientes com úlcera arterial, dois com úlcera venosa e um com pé diabético. Constatou-se que o Formulário de Avaliação de Feridas (Figura 1) não constava nos prontuários e, portanto, não estava sendo usado para seguimento das feridas. Além disso, os folhetos informativos (Figura 2) direcionados aos pacientes de risco também não foram distribuídos. A equipe da UAPS informou que não foi possível a implantação do formulário e a distribuição dos folhetos devido à mudança na composição da equipe.

Em entrevistas realizadas com a equipe responsável diretamente pelos cuidados com feridas crônicas, foi implicada como principal dificuldade no cuidado a falta de material adequado. Outros fatores citados foram: dificuldade em caracterizar o estágio de cicatrização da ferida, falta de adesão ao tratamento dos pacientes (principalmente o não-medicamentoso) e no caso da ESF Nova Aliança, a grande distância de algumas áreas abrangidas em relação a UAPS, o que dificulta o seguimento de alguns pacientes. 
ISSN 2179-6750

Tabela 1. Dados das pessoas com feridas crônicas acompanhadas pela atenção primária à saúde (entre agosto de 2013 e agosto de 2014)

\begin{tabular}{|c|c|c|c|c|c|c|c|c|c|c|c|}
\hline \multicolumn{3}{|c|}{ Pacientes } & \multicolumn{3}{|c|}{$\begin{array}{l}\text { Qualidade do registro } \\
\text { no prontuário }\end{array}$} & \multicolumn{2}{|c|}{ Ferida } & \multicolumn{2}{|c|}{$\mathbf{N}^{\circ}$ de consultas } & \multicolumn{2}{|c|}{$\begin{array}{l}\mathbf{N}^{\circ} \text { de visitas } \\
\text { domiciliares }\end{array}$} \\
\hline $\mathbf{N}^{\circ}$ & Sexo & Idade & $\begin{array}{l}\text { Folha } \\
\text { de } \\
\text { rosto }\end{array}$ & $\begin{array}{l}\text { Lista de } \\
\text { problemas }\end{array}$ & SOAP* & $\begin{array}{l}\text { Etiologia } \\
\text { da úlcera }\end{array}$ & $\begin{array}{c}\text { Infecções } \\
\text { no último } \\
\text { ano }\end{array}$ & Médico & Enfermagem & Médico & Enfermagem \\
\hline 1 & $\mathrm{M}$ & 60 & $\mathrm{x}$ & & & Arterial & 0 & 2 & 1 & 4 & 1 \\
\hline 2 & $\mathrm{M}$ & 48 & $\mathrm{x}$ & & & Arterial & 1 & 7 & 3 & 0 & 0 \\
\hline 3 & $\mathrm{~F}$ & 78 & $\mathrm{x}$ & $\mathrm{x}$ & & $\begin{array}{c}\text { Pé } \\
\text { diabético }\end{array}$ & 0 & 3 & 1 & 4 & 4 \\
\hline 4 & M & 74 & $\mathrm{x}$ & $\mathrm{x}$ & $\mathrm{x}$ & $\begin{array}{c}\text { Neoplási } \\
\text { ca }\end{array}$ & 3 & 6 & 0 & 11 & 21 \\
\hline 5 & $\mathrm{~F}$ & 62 & $\mathrm{x}$ & $\mathrm{x}$ & $\mathrm{x}$ & Venosa & 1 & 6 & 2 & 0 & 0 \\
\hline 6 & $\mathrm{M}$ & 63 & $\mathrm{x}$ & $\mathrm{x}$ & $\mathrm{x}$ & Venosa & 1 & 12 & 17 & 0 & 0 \\
\hline 7 & $\mathrm{M}$ & 58 & $\mathrm{x}$ & $\mathrm{x}$ & $\mathrm{x}$ & Venosa & 0 & 0 & 0 & 12 & 20 \\
\hline 8 & $\mathrm{~F}$ & 63 & $\mathrm{x}$ & $\mathrm{x}$ & $\mathrm{x}$ & Venosa & 1 & 6 & 10 & 0 & 0 \\
\hline 9 & M & 67 & $\mathrm{x}$ & $\mathrm{X}$ & $\mathrm{x}$ & $\begin{array}{l}\text { Venosa/ } \\
\text { Arterial }\end{array}$ & 0 & 1 & 2 & 2 & 4 \\
\hline 10 & $\mathrm{~F}$ & 77 & & & & Venosa & 1 & 1 & 0 & 4 & 5 \\
\hline 11 & $\mathrm{~F}$ & 44 & $\mathrm{x}$ & $\mathrm{x}$ & $\mathrm{x}$ & Venosa & 0 & 0 & 0 & 2 & 2 \\
\hline 12 & $\mathrm{M}$ & 62 & $\mathrm{x}$ & $\mathrm{x}$ & & Venosa & 4 & 13 & 13 & 1 & 1 \\
\hline 13 & M & 49 & & & & Venosa & 0 & 4 & 25 & 0 & 0 \\
\hline 14 & $\mathrm{~F}$ & 90 & $\mathrm{x}$ & $\mathrm{x}$ & $\mathrm{x}$ & Pressão & 1 & 4 & 0 & 18 & 23 \\
\hline 15 & $\mathrm{M}$ & 58 & $\mathrm{x}$ & $\mathrm{x}$ & $\mathrm{x}$ & Pressão & 0 & 0 & 0 & 10 & 4 \\
\hline 16 & $\mathrm{~F}$ & 63 & & & $\mathrm{x}$ & Venosa & 1 & 1 & 0 & 0 & 0 \\
\hline 17 & $\mathrm{~F}$ & 72 & $\mathrm{x}$ & $\mathrm{x}$ & $\mathrm{x}$ & $\begin{array}{c}\text { Pé } \\
\text { diabético }\end{array}$ & 1 & 2 & 9 & 7 & 4 \\
\hline
\end{tabular}

* Registro orientado ao problema

\section{Conclusão}

O cuidado das pessoas com feridas, sejam elas crônicas ou agudas, é responsabilidade da Atenção Primária a Saúde ${ }^{9}$. O autocuidado é fator determinante no surgimento, cronificação e cura de feridas crônicas, relacionando-se a variáveis como condições sócio-economica-cultural das pessoas, de suas família e de sua comunidade; hábitos de higiene, literacia sobre o feridas crônicas, acesso aos serviços de saúde (recursos humanos e insumos para tratamento) e estrutura familiar e comunitária. As comorbidades mais comumente associadas as feridas cronicas são as doenças crônicas não transmissíveis (HAS e DM) e o fator de risco dislipidemia. O tema "feridas" ainda possui mitos relacionados ao processo de cura e traz ganhos pessoais secundários, dificultando a adesão ao tratamento recomendado. A implementação de estratégias de prevenção e de assistência ao portador de feridas (ex.: aumento da literacia em saúde e empoderamento do paciente, otimização do registro e da assistência prestada pela ESF) podem impactar positivamente a qualidade de vida do paciente, a sistematização do trabalho dos profissionais de saúde e o sistema local de saúde. Entretanto, maior ênfase no desenho e na implementação da intervenção com participação ampla dos profissionais de saúde desde o início se faz necessária. 


\section{Referências}

1. Oliveira BGRB, Lima FFS, Araujo JO. Ambulatório de reparo de feridas - perfil da clientela com feridas crônicas. Um estudo prospectivo. Online Braz J Nurs. 2008; 7(2): 1-4.

2. Miot HA, Mendaçolli TJ, Costa SV, Haddad GR, Abbade LPF. Úlceras crônicas dos membros inferiores: avaliação pela fotografia digital. Rev Assoc Med Bras. 2009; 55(2):145-8.

3. Hughes C, Smith M, Tunney M. Infection control strategies for preventing the transmission of meticillin-resistant Staphylococcus aureus (MRSA) in nursing homes for older people. Cochrane Database Syst Rev, 2011(12): 1-4.

4. Lucas LS, Martins JT, Robazzi MLCC. Qualidade de vida dos portadores de ferida em membros inferiores - úlcera de perna. Cienc Enferm. 2008; 14 (1): 43-52.

5. Eccles MP, Weijer C, Mittman B. Requirements for ethics committee review for studies submitted to Implementation Science. Implement Sci. 2011; 6(32):1-3.

6. Vu T, Harris A, Duncan G, Sussman G. Cost-effectiveness of multidisciplinary wound care in nursing homes: a pseudo-randomized pragmatic cluster trial. Fam Pract. 2007; 24(4): 37279.

7. Sanada H, Nakagami G, Mizokami Y, Minami Y, Yamamoto A, Oe M, et al. Evaluating the effect of the new incentive system for high-risk pressure ulcer patients on wound healing and cost-effectiveness: a cohort study. Int J Nurs Stud. 2010; 47(3): 279-86.

8. Tramontini SJ, Peruzzo AB. Cuidado com feridas. In: Gusso G, Lopes JMC. Tratado de Medicina de Família e Comunidade. Princípios, Formação e Prática. Porto Alegre: Artmed; 2012. p.1566-74.

9. Caramori JE; Longhi EG. Necrose e abscesso na região posterior da coxa. Rev Bras Med Fam Comunidade. 2010; 3 (12): 299-305. 